\title{
Crisis económica y mortalidad infantil en Latinoamérica desde los años ochenta
}

\author{
Economic crisis and infant mortality \\ in Latin America since 1980
}

Dalia E. Romero 1

Célia Landmann Szwarcwald 1

1 Departamento de
Informações em Saúde,
Centro de Informação
Científica e Tecnológica,
Fundação Oswal do Cruz.
Av. Brasil 4365,
Rio de Janeiro, RJ
$21041-210$, Brasil.
dalia@fiocruz.br
celia@malaria.procc.fiocruz.br

\begin{abstract}
In the present study, based on data from nine Latin American countries, we found evidence of an association between the economic crisis and infant mortality during the last decades. The paper initially review previous studies on this issueand shows the need for a greater research focus on shorter time intervals. We then describe the deterioration and unequal conditions among the countries based on trends in sel ected social and economic indicators and the evolution of infant mortality rates. According to our statistical analysis, infant mortality bore an inverse associati on to short-term economic variations. We also found a significant and negative correlation between decreasing infant mortality rates and increasing poverty. The economic crisis displayed effects of varying intensity among the countries we analyzed, with social inequality appearing as the most probable explanatory variable.

Key words Infant Mortality; Poverty; Economics; Latin America
\end{abstract}

Resumen En este trabajo realizado con información de nueve países latinoamericanos, se encontraron evidencias de la asociación entre crisis económica y mortalidad infantil durantelas últimas décadas. En primer lugar, se hace referencias a los aportes anteriores relaci onados con el tema y se constata la necesi dad de más investigaciones con períodos de corto plazo. De seguido, se describe el deterioro y las desigual dades entre los países según la evol ución de los indicadores socio-económicos sel eccionados y la evol ución del coci ente de mortali dad infantil. Los hallazgos del análisis estadístico muestran que la mortalidad infantil está asociada negativamente con mudanzas económi cas de corto plazo. Se observó correlación negativa y si gni ficativa entre el ritmo de decrecimiento del CMI y el crecimiento dela pobreza. El impacto dela crisis económica de los ochenta tuvo diferente intensi dad entre los países consi derados, si endo que la desi gualdad del ingreso aparece como una de las explicaciones más plausibles.

Palabras clave Mortalidad Infantil; Pobreza; Economía; Latinoamérica 


\section{Introdución}

El estudio de la relación entre las condiciones económicas y la mortalidad es de larga data. Especialmente en América Latina se encuentran importantes estudios que demuestran la transición de la mortalidad y su relación con el tipo de desarrollo socioeconómico establecido (Behm, 1979; Breilh \& Granda, 1984; Chackiel, 1984). Sin embargo, es poco lo que se ha escrito acerca de los efectos que tienen las crisis económicas recientes sobre la mortalidad infantil en un período corto como una o dos décadas, sobre todo si se compara con lo que se conoce acerca del período pre-industrial .

Palloni y Tienda (1992) señalan dos explicaciones para lo anterior. En primer lugar, una implícita (y probablemente errónea) creencia de que, con pocas excepciones, los patrones demográficos en sociedades modernas en desarrollo son altamente inmunes a fluctuaciones económicas de corto plazo. Inclusive, algunos observadores afirman que, a partir de los años cuarenta, las dramáticas ganancias experimentadas en la salud marcan el comienzo de un período en el cual respuestas demográficas para desviaciones transitorias de tendencias económicas seculares son apenas tenues y de poco interés.

La escasez de estadísticas confiables y oportunas es otra razón para explicar el por qué de haber pocos estudios sobre América Latina que den cuenta del impacto de la crisis económica en eventos socio-demográficos como la mortalidad infantil. El trabajo de investigación requiere de estadísticas, las cuales, en países no desarrollados, generalmente son defectuosas, problema que se incrementa cuando el foco de interés es un período corto. Por otra parte, porque la tentativa para establecer asociaciones, sobre todo relaciones causales, tienen frecuentemente poco suceso, muchos investigadores prefieren tendencias de largo plazo (ya que las estadísticas a largo plazo dan mayor seguridad de estimaciones relativamente robustas) a aquellos análisis detallados de series de tiempo que permitan sólo débiles inferencias acerca de cambios de corto plazo.

Sin pretender ser exhaustivo, se puede afirmar que, entre los estudios de más referencia que tratan del efecto de la reciente crisis económica en las condiciones de salud, se encuentran los de Palloni (1990), Palloni \& Tienda (1992), Hill \& Palloni (1994), Bravo \& Rodriguez (1993). Esos estudios pasaron a ser referencias importantes en el tema por la diversidad de países latinoamericanos estudiados y principalmente por la metodología y conclusiones alcanzadas. Los autores retoman los modelos de distribución de atraso (distributed lag models) para efectos a corto plazo (hasta cuatro años de rezago). Ya estudios en países específicos son más frecuentes, tales como el de Bravo (1997) sobre Chile, Bronfman (1992) sobre México y Ríos-Neto \& Carvalho (1997) sobre Brasil.

Como lo señalan Bajraj \& Bravo (1993), dos reuniones de carácter internacional pueden ilustrar tal preocupación en América Latina: una sesión especial de la Conferencia sobre Poblamiento de las Américas en Veracruz (México, 1992) y un seminario patrocinado por la Unión Internacional de Estudios de Población (UIECP) en Ouro Preto (Brasil), en octubre de 1992. Ambas reúnen interesantes evidencias empíricas y algunas propuestas metodológicas. Los trabajos de este último resultaron en un libro editado por Tapinos, Mason \& Bravo (1997).

Existe cierto consenso en cuanto a las diversas dificultades para precisar cual es la relación entre tendencias económicas y eventos como la mortalidad infantil, la nupcialidad, la natalidad, entre otros. Uno de esos problemas es el relacionado con la temporalidad de los eventos. De las cuestiones más polémicas en ese sentido se refiere al período de tiempo que se debe esperar para que una crisis económica haga efecto sobre los eventos socio-demográficos. Como lo señalan Hill \& Palloni (1994), una alternativa sería estudiar los años de rezago, es decir, en cuantos años de diferencia entre el indicador económico y la variable demográfica estudiada se da el mayor efecto.

El segundo problema, y no menos importante, es cómo reconocer ese impacto. Existe la cuestión en cuanto a lo qué se debe esperar frente a una crisis: una relación inversa y directa ó una diversidad de impactos, que pueden ser mediados por políticas sociales compensatorias, programas, condiciones políticas, entre otros. Conforme sugerido por Musgrove (1997), para observar esa mediación, se recomienda incorporar en el análisis las condiciones institucionales, políticas y de organización social.

El continuo descenso del Cociente de Mortalidad Infantil (CMI) en la mayor parte de los países de América Latina, en períodos de crisis económica, no significa que sean eventos independientes (Bajraj \& Bravo, 1993). Se puede dar, como en el caso de Brasil en la década de los ochenta, una disminución del CMI junto con una ampliación de la desigualdad social, mas con una tasa de decrecimiento del CMI que no se mantuvo constante (Szwarcwald et al., 1997). Oliveira \& Simões (1986) y Bravo (1997) mencionan el papel de las políticas públicas en los cambios de las condiciones de vida de la 
población como una condición importante para la reducción del CMI, mas que, sin embargo, aquellas no consiguieron disminuir las grandes brechas de desigualdad social ante la muerte.

Como se demuestran en estudios sobre experiencia brasileña (Wood \& Carvalho, 1988), cuando se tienen períodos de empobrecimiento económico, los indicadores agregados muestran mejoras de los CMI, mas una simple desagregación puede revelar que grupos sociales están negativamente afectados, mientras que otros grupos incrementan su prosperidad en los peores años de crisis económica.

Por otra parte, a pesar de que la desigualdad social y el deterioro del ingreso (sea individual o familiar) han sido dos de las principales consecuencias directas del proceso de ajuste estructural aplicado, desde los años ochentan en la gran mayoría de países latinoamericanos (Petras \& Veltmeyer, 1995), se puede afirmar que no se los han incorporado de forma suficiente en los estudios que intentan explicar el impacto en la mortalidad infantil de los cambios iniciados desde 1980, con la gradual implantación de la política neoliberal. Esa carencia de estudios queda más evidenciada cuando se toma en cuenta que estudios ecológicos han demostrado (Townsend \& Davidson, 1990; Wilkinson, 1992) que la asociación entre renta y salud no es de forma lineal; en otras palabras, las diferencias en la renta no corresponden con la misma intensidad y dirección a cambios en los indicadores de salud. Townsend \& Davidson (1990), encontraron que, a pesar de la disminución de la pobreza absoluta ocurrida en Gran Bretaña, las diferencias en las condiciones de salud entre los diferentes estratos sociales no sólo persistieron sino que se agravaron. En ese sentido, recomiendan utilizar indicadores de renta relativa, en lugar de renta absoluta, cuando se estudia la desigualdad relativa a un contexto socio-espacial.

De las recomendaciones más relevantes de los estudios mencionados anteriormente se tiene que, para reconocer el impacto de la situación económica en la mortalidad infantil, es necesario conocer cómo es la situación demográfica del lugar (estructura de edad, distribución de la población, vulnerabilidad de la población y nivel de la fecundidad) (Palloni, 1994). Así mismo, es importante tener escenarios para comparar con respecto a un valor esperado, bien sea con respecto a la tendencia histórica de los eventos o a otro patrón sometido a diferentes condiciones socio-económicas (Bajraj \& Bravo, 1993).

La década de los años ochenta ha sido calificada como "década perdida", para destacar los atrasos no sólo económicos sino sociales que se dieron en la región latinoamericana. Mas es importante realizar reflexiones en torno a tal concepto. Si bien se observó una "recuperación moderada" de indicadores macroéconómicos a principios de los años ochenta, no puede dejar de señalarse que respondieron a una seria imposición de políticas neoliberales de alto costo social. Como señalan Petras \& Veltmeyer (1995), la profunda crisis económica que América Latina experimentó en la década de los años ochenta sentó las bases para cambios importantes en el sistema político económico. La recuperación de indicadores macroeconómicos en el comienzo de los años noventa, como respuesta al marco de políticas neoliberales implantadas en la región, significó una serie de pérdidas en relación a la calidad de vida, debido, en gran parte, a la instauración de cambios estructurales en el orden la relación capital-trabajo, en el papel del Estado y en la estructura interna del capital (Petras \& Veltmeyer, 1995). Algunas de las principales consecuencias de esos cambios estructurales, las cuales se agudizan hasta hoy, son el creciente nivel de desempleo, el debilitamiento y hasta destrucción de las organizaciones laborales, la depresión de los salarios y el aumento consecuente de la pobreza.

El impacto de esas medidas económicas, implantadas desde los años ochenta, en las condiciones socio-demográficas de cada país depende, en gran medida, de las posibilidades políticas de implantación de las medidas de austeridad impuestas por agencias internacionales y de la severidad del impacto doméstico de la administración de la deuda y de las políticas de estabilización (Cornia, 1984; Palloni \&Tienda, 1992).

En muchos países, esas medidas han llevado al corte drástico del gasto social (de subsidios de alimentos, educación, salud y otros). A nivel interno, el gobierno y las fuerzas económicas nacionales pueden reducir el número de empleos, los programas públicos y la ayuda para programas sociales. Con ello, pueden verse afectadas negativamente las unidades de atención primaria de salud, los programas nutricionales y de atención materno-infantil.

Aunque, por la carencia de información, no sea posible constatar a nivel empírico el conjunto de interrelaciones entre las variables socio-económicas que caracterizaron la crisis de los años ochenta y el resultado de la mortalidad infantil, en este trabajo se intenta la búsqueda de comprensión de los distintos comportamientos de los CMI en América Latina, desde el comienzo de la llamada "década perdida", a través de las variables disponibles. 
A pesar de que no se trate de explicar los determinantes de los niveles del CMI en los diferentes países latinoamericanos ni de buscar determinar el número de años entre la crisis económica y sus efectos en el CMI, se procura identificar los indicadores socio-económicos más correlacionados con las variaciones temporales en el CMI, durante las últimas dos décadas.

\section{Materiales y método}

Para la selección de los países a incluir en este trabajo, se tomó como referencia el Indice de Desarrollo Humano (IDH) de las Naciones Unidas (Naciones Unidas, 1998). Para comparar los que guardasen cierta semejanza en el nivel de desarrollo al canzado, los países seleccionados son los latinoamericanos calificados como pertenecientes al grupo de al to Indice de Desarrollo Humano, los cuales, en orden, son: Chile (en el puesto 31), Costa Rica (34), Argentina (36), Uruguay (38), Panamá (45), Venezuela (46), México (49), Colombia (53) y Brasil (62). Tales países poseen mejor sistema de información que el resto de los países latinoamericanos. Sin embargo, también padecen, en diferente medida, de problemas de cobertura, disponibilidad y acceso a la información. Por ello, no es posible construir una serie de datos completas por año calendario, ni tener información para todas las variables en el mismo período.

A continuación, se presentan los indicadores usados en este estudio, así como las fuentes de información usadas para su obtención.

1) Los indicadores demográficos CMI y la Tasa Global de Fecundidad (TGF) se tomaron de las estimaciones realizadas por el Centro Latinoamericano de Demografía (CELADE, 1996) por ser el organismo oficial de Naciones Unidas a ese respecto. EI CELADE corrige la calidad y la cobertura de información aplicando el método de proyección de población.

2) El Cociente de Mortalidad Infantil por Causas Infecciosas se estimó a partir de las defunciones por causas publicadas por las Estadísticas de Salud de las Américas de la Organización Panamericana de la Salud (OPS, 1995), en relación a los nacimientos estimados por el CELADE (1996).

3) A partir de las estadísticas publicadas por el Banco Mundial en sus informes sobre Desarrollo (World Bank, 1991, 1996), se obtienen:

- El Crecimiento interanual del Producto Interno Bruto (PIB) por habitante, el cual define las tasas anuales medias sobre la base de cifras a precios constantes de 1990
- La deuda externa total como porcentaje del Producto Nacional Bruto (PNB).

- Crecimiento Interanual del PIB. Se refiere a la tasa media de crecimiento anual en porcentaje.

4) Las variables que se recolectan de los Anuarios Estadísticos de la Comisión Económica para América Latina y el Caribe (CEPAL, 1990, 1995) son:

- Porcentaje de Analfabetos. Se refiere a la población analfabeta mayor de 15 años.

- El Gasto en Salud, calculado como el porcentaje del gasto publico en salud del gobierno central consolidado en el PIB a precios corrientes de mercado, excluyendo lo que no es del gobierno central.

- El porcentaje de población en area metropolitana, definido como el número de habitantes en la superficie de la ciudad central, más las zonas de alta densidad de su entorno.

- Porcentaje del ingreso del 10\% de hogares más ricos. Se refiere al decil 10 de la distribución del ingreso de los hogares urbanos, ordenados según el ingreso per cápita.

- La desigualdad del ingreso entre los más ricos y los más pobres se estimó como el cociente del total del ingreso per cápita del $10 \%$ de los hogares urbanos más ricos entre el ingreso per cápita que acumula el $20 \%$ de los hogares urbanos más pobres.

- El indicador de pobreza urbana se refiere al porcentaje de hogares cuyo ingreso es inferior al doble de una canasta básica de alimentos. Incluye los hogares en situación de indigencia (ingreso menor a una canasta básica). Las estimativas para los años no siempre se refieren al año exacto, sino a un año antes u otro posterior al señalado. Se seleccionó la unidad urbana por la mayor disponibilidad de información.

- Consumo Privado por Habitante. Está estimado a partir de las tasas medias anuales sobre la base de cifras a precios constantes de 1990.

Se estiman el desvío padrón y la media de los indicadores para el conjunto de todos los países latinoamericanos estudiados. Se considera cada país con el mismo peso debido a que, con la ponderación, el resultado de la media reflejaría especialmente aquellos países con mayor población, tales como México y Brasil. Por tanto, con tales indicadores estudiados, no se pretende representar a la región latinoamericana.

Se seleccionó la información en tres momentos diferentes de la historia reciente de los cambios socioeconómicos de América Latina, siendo ellos el comienzo de las tres últimas décadas. Como no se encuentra disponible, para la mayoría de las variables, la información para 
cada año, se estableció el criterio de la disponibilidad y representatividad del año calendario para un período. Como no todas las variables tienen el mismo ritmo de modificación algunas variables se toman para el período quinquenal y otras para un año puntual. En el caso del CMI y de la TGF, se toma por períodos quinquenales, para así observar el momento de mayor transición demográfica en tal período. El gasto en salud, los datos relacionados con ingreso y el porcentaje de pobreza estan disponibles para la mayoría de los países considerados sólo a partir de 1980. El crecimiento interanual del PIB se toma entre 1965 y 1980 y entre 1980 y 1989, para comparar el deterioro económico en la llamada década perdida y los años anteriores.

Para analizar los datos, primero se describen las tendencias de los indicadores seleccionados y, a continuación, se presenta un análisis estadístico de las correlaciones entre el CMI y las variables seleccionadas en periodos anteriores y similares. Debido al número pequeño de países incluidos en el estudio, considerase 10\% como nivel crítico de significancia estadística.

Ese análisis también se realiza comparando las variaciones porcentuales de los indicadores en períodos determinados de tiempo que representen de alguna manera el anterior (o comienzo) y el auge de la crisis económica desde los ochenta. Ello con la finalidad de reconocer la asociación con la intensidad de la reducción del CMI en la región.

\section{Resultados}

Apreciación de los indicadores socioeconómicos utilizados

La intensa crisis económica durante la década de los ochenta es reflejada en diversos índices. En la Tabla 1 se observa que, durante los años que van de 1980 a 1987, la tasa acumulada de crecimiento del PIB por habitante fue negativa en casi todos los países latinoamericanos considerados para la comparación, exceptuando a Brasil y Colombia con crecimiento casi nulo. El descenso medio fue de al rededor de $20 \%$ durante esa década, resultado muy influenciado por Venezuela, el cual extrapoló ese promedio, llegando a alrededor de $160 \%$ el descenso acumulado.

Notase también que lo destinado del PNB para el pagamento de la deuda aumenta, en media, para los países latinoamericanos considerados, de 33\% para 71\% entre 1976 y 1986. México y Venezuela son los países que más aumentaron su endeudamiento en ese período, en tres y cuatro veces, respectivamente. Es en esos dos países donde el salario real sufre el mayor deterioro, entre 1979 y 1987 (CEPAL, 1995).

Un indicador que refleja el efecto de la crisis económica en los hogares es el consumo privado por habitante. Con el se refleja claramente la pérdida en la calidad de vida del latinoamericano entre la década de los años setenta y la de los ochenta. M ientras que, en los años setenta, la tendencia fue de crecimiento del consumo privado en todos los países (de 6,2 en Brasil a 0,3 en Chile), en la década de lo ochenta fue de un decrecimiento acentuado. En promedio, para la región, los hogares reducen en casi dos veces el consumo entre 1980 y 1990 . El de mayor deterioro en ese sentido es Venezuela (menos de cinco veces el consumo de 1990 en relación al de 1980), seguido de Argentina y Chile.

Como contrapartida a esa disminución del consumo privado y deterioro económico, está el empobrecimiento acelerado de los hogares latinoamericanos. El alto índice de pobreza urbana ya al canzado a finales de los setenta (en promedio 2 de cada 10 hogares de la región) aumenta progresivamente durante los años ochenta en la región. Mismo en Argentina, con el menor índice de hogares pobres en 1994, esa proporción es casi cuatro veces lo que representaba en 1980. En Chile, el crecimiento fue de tres veces (aunque, desde 1986, muestre una tendencia a la reducción de la pobreza), mientras que, en Venezuela y M éxico, casi se duplicó el nivel de pobreza al Ilegar a 1994. En Colombia y Brasil, la pobreza está ya en altos niveles desde comienzos de la década de los ochenta, países en donde al rededor de 4 hogares de cada 10 son calificados como pobres.

El porcentaje del ingreso acumulado por el $10 \%$ de los hogares más ricos no se vio desfavorecido por la crisis económica sino que, por el contrario, aumentó sistemáticamente esa acumulación (pasando de ser 31\% en 1980 a 35\% en 1994). Llama la atención que es justamente en el primer quinquenio de los ochenta cuando más se acelera la crisis económica y la velocidad del crecimiento de la concentración del ingreso en pocos hogares. En 1980, en promedio, una familia de cada diez se apodera de la tercera parte de la riqueza nacional. De esa data a 1994, solamente en Uruguay y Colombia se mantuvo relativamente estable la proporción de la riqueza acumulada. A pesar de Venezuela, en 1994, continuar siendo uno de los países con menor acumulación del ingreso en el $10 \%$ de hogares más ricos (perdiendo sólo de Uruguay y Costa Rica), es el de mayor velocidad de crecimiento de esa acumulación (aumenta $44 \%$ en el período señalado, es decir, el 10\% de los más 
Tabla 1

Indicadores socio-económicos de países latinoamericanos 1970-1994.

\begin{tabular}{lccccccccccccc}
\hline IDH & Chile & Costa Rica & Argentina & Uruguay & Panamá & Venezuela & México & Colombia & Brasil & Media & Desvio P. \\
\hline 31 & 34 & 36 & 38 & 45 & 46 & 49 & 53 & 62 & 43,8 & 10,0
\end{tabular}

Cociente de mortalidad

infantil (por 1.000

nacidos vivos)

\begin{tabular}{|c|c|c|c|c|c|c|c|c|c|c|c|}
\hline $1970-75$ & 69,9 & 52,6 & 49,0 & 46,3 & 42,8 & 48,6 & 70,9 & 73,0 & 90,5 & 60,4 & 16,2 \\
\hline $1975-80$ & 45,1 & 30,3 & 39,0 & 42,3 & 35,3 & 39,2 & 56,7 & 59,3 & 78,5 & 47,3 & 15,0 \\
\hline $1980-85$ & 23,7 & 19,2 & 32,1 & 33,4 & 30,4 & 33,5 & 46,9 & 41,0 & 64,2 & 36,0 & 13,4 \\
\hline $1985-90$ & 18,3 & 16,0 & 27,1 & 24,4 & 28,4 & 26,8 & 39,4 & 34,5 & 54,8 & 30,0 & 11,8 \\
\hline 1990-95 & 14,0 & 13,7 & 24,3 & 19,9 & 25,1 & 23,1 & 33,9 & 28,0 & 47,0 & 25,4 & 10,3 \\
\hline
\end{tabular}

Tasa global de fecundidad

\begin{tabular}{|c|c|c|c|c|c|c|c|c|c|c|}
\hline $1970-75$ & 3,6 & 4,3 & 3,2 & 3,0 & 4,9 & 4,9 & 6,5 & 4,7 & 4,7 & 4,4 \\
\hline $1975-80$ & 3,0 & 3,9 & 3,4 & 2,9 & 4,1 & 4,5 & 5,3 & 4,1 & 4,3 & 3,9 \\
\hline $1980-85$ & 2,7 & 3,5 & 3,2 & 2,6 & 3,5 & 4,0 & 4,2 & 3,4 & 3,6 & 3,4 \\
\hline $1985-90$ & 2,7 & 3,4 & 3,0 & 2,4 & 3,2 & 3,6 & 3,6 & 3,2 & 3,0 & 3,1 \\
\hline 1990-95 & 2,5 & 3,1 & 2,8 & 2,3 & 2,9 & 3,3 & 3,1 & 2,9 & 2,4 & 2,8 \\
\hline
\end{tabular}

Cociente de mortalidad

infantil por causas

infecciosas (por 1.000

nacidos vivos)

\begin{tabular}{|c|c|c|c|c|c|c|c|c|c|c|}
\hline 1970 & 14,2 & 14,6 & 7,8 & 4,2 & 4,8 & 8,9 & 15,7 & 9,4 & - & 9,9 \\
\hline 1980 & 2,0 & 1,3 & 2,5 & 3,7 & 1,4 & 4,3 & 9,7 & 9,0 & 8,6 & 4,7 \\
\hline 1990 & 0,4 & 0,8 & 0,9 & 0,9 & 1,0 & 2,8 & 4,2 & 1,7 & 3,9 & 1,8 \\
\hline
\end{tabular}

Porcentaje de analfabetismo

$\begin{array}{rrrrrrrrrrrr}1970 & 11,0 & 11,6 & 7,4 & 6,1 & 18,7 & 23,5 & 25,8 & 19,2 & 33,8 & 17,5 & 9,2 \\ 1980 & 8,9 & 7,4 & 6,1 & 5,0 & 12,9 & 15,3 & 16,0 & 12,2 & 25,5 & 12,1 & 6,4 \\ 1990 & 5,7 & 7,2 & 4,0 & 3,8 & 11,2 & 10,2 & 12,4 & 13,3 & 18,3 & - & -\end{array}$

Porcentaje de población

en área metropolitana

$\begin{array}{rrrrrrrrrrrr}1970 & 32,3 & 20,8 & 35,6 & 48,2 & 31,7 & 20,3 & 18,5 & 14,0 & 8,4 & 25,5 & 12,4 \\ 1980 & 34,8 & 19,8 & 35,7 & 49,5 & 35,1 & 18,1 & 20,8 & 14,8 & 10,2 & 26,5 & 12,8 \\ 1990 & 35,4 & 20,0 & 34,5 & 41,6 & 36,3 & 15,3 & 18,5 & 15,8 & 10,6 & 25,3 & 11,5\end{array}$

Gasto salud (\% PIB)

\begin{tabular}{|c|c|c|c|c|c|c|c|c|c|c|}
\hline 1980 & 2,1 & 7,2 & 1,6 & 1,0 & 1,6 & 1,3 & 0,4 & 1,2 & 1,3 & 2,0 \\
\hline 1985 & 2,7 & 5,0 & 1,6 & 1,0 & 1,8 & 1,8 & 0,3 & 1,2 & 2,1 & 1,9 \\
\hline 1990 & 2,0 & 6,3 & 4,4 & 1,4 & 1,7 & 1,3 & 0,4 & 1,3 & 2,9 & 2,4 \\
\hline 1995 & 2,5 & 7,1 & 4,8 & 1,6 & 2,4 & 1,5 & & 3,8 & 2,8 & 3,3 \\
\hline
\end{tabular}

Crecimiento del consumo privado por habitante

\begin{tabular}{|c|c|c|c|c|c|c|c|c|c|c|c|}
\hline $1970-80$ & 0,3 & 1,8 & 1,4 & 2,0 & 1,3 & 5,0 & 2,5 & 3,2 & 6,2 & 2,6 & 1,9 \\
\hline $1980-90$ & $-1,1$ & 0,0 & $-1,5$ & $-0,5$ & 1,0 & $-5,6$ & 0,3 & 1,1 & 0,1 & $-0,7$ & 2,0 \\
\hline
\end{tabular}


Tabla 1 (continuación)

\begin{tabular}{|c|c|c|c|c|c|c|c|c|c|c|c|}
\hline IDH & Chile & Costa Rica & Argentina & Uruguay & Panamá & Venezuela & México & Colombia & Brasil & Media & Desvio P. \\
\hline \multicolumn{12}{|c|}{$\begin{array}{l}\text { Porcentaje del ingreso del } \\
10 \% \text { de hogares más ricos }\end{array}$} \\
\hline 1980 & - & 23,2 & 30,9 & 31,2 & 29,1 & 21,8 & - & 41,3 & 39,1 & 30,9 & 7,3 \\
\hline 1986 & 39,6 & 27,6 & 34,5 & 33,6 & 33,0 & 28,9 & 25,8 & 35,3 & 44,3 & 33,6 & 5,8 \\
\hline 1990 & 39,2 & 24,6 & 34,8 & 31,2 & 36,2 & 28,4 & 36,9 & 34,9 & 41,7 & 34,2 & 5,3 \\
\hline 1994 & 40,4 & 27,6 & 34,2 & 25,4 & 37,4 & 31,4 & 34,3 & 41,9 & 42,5 & 35,0 & 6,1 \\
\hline \multicolumn{12}{|c|}{$\begin{array}{l}\text { Desigualdad del ingreso } \\
\text { ( } 10 \% \text { más ricos } / 20 \% \\
\text { más pobres) }\end{array}$} \\
\hline 1980 & 3,4 & 4,5 & 4,6 & 6,2 & 3,2 & - & 12,1 & 10,0 & 6,3 & 3,5 & - \\
\hline 1986 & 9,0 & 4,3 & 5,4 & 4,5 & 7,7 & 5,2 & 3,3 & 9,1 & 14,8 & 7,0 & 3,6 \\
\hline 1990 & 8,3 & 4,3 & 5,6 & 3,8 & 8,6 & 5,0 & 6,0 & 7,6 & 13,5 & 7,0 & 3,0 \\
\hline 1994 & 8,6 & 4,8 & 6,8 & 2,9 & 8,1 & 5,1 & 5,0 & 11,3 & 10,6 & 7,0 & 2,9 \\
\hline \multicolumn{12}{|c|}{ Porcentaje de hogares pobres } \\
\hline 1970 & 12 & 15 & 5 & 10 & - & 20 & 20 & 38 & 35 & 19,4 & 11,7 \\
\hline 1980 & 41 & 16 & 7 & 9 & 31 & 18 & 20 & 36 & 30 & 23,1 & 11,9 \\
\hline 1986 & 37 & 21 & 12 & 14 & 28 & 25 & 28 & 36 & 34 & 26,1 & 9,1 \\
\hline 1990 & 34 & 22 & 19 & 10 & 34 & 33 & 34 & 35 & 39 & 28,9 & 9,6 \\
\hline \multicolumn{12}{|c|}{$\begin{array}{l}\text { Crecimiento interanual del } \\
\text { producto interno bruto }\end{array}$} \\
\hline $1965-1980$ & 1,9 & 6,3 & 3,4 & 2,4 & 5,5 & 3,7 & 6,5 & 5,7 & 9 & 4,9 & 2,3 \\
\hline 1980-1989 & 2,7 & 2,8 & $-0,3$ & 0,1 & 0,5 & 1 & 0,7 & 3,5 & 3 & 1,6 & 1,4 \\
\hline \multicolumn{12}{|c|}{$\begin{array}{l}\text { Tasa acumulada de } \\
\text { crecimiento del PIB } \\
\text { en } \$ \text { U.S.de } 1986\end{array}$} \\
\hline 1980-87 & $-0,03$ & $-0,10$ & $-0,14$ & $-0,09$ & $-0,04$ & $-1,66$ & $-0,10$ & 0,07 & 0,03 & $-0,2$ & 0,5 \\
\hline \multicolumn{12}{|c|}{$\begin{array}{l}\text { Deuda externa total como } \\
\text { porcentual del PNB }\end{array}$} \\
\hline 1976 & 57 & 40,8 & 18,1 & 28,4 & 68,7 & 13,2 & 25,1 & 25,7 & 21,8 & 33,2 & 18,7 \\
\hline 1986 & 119,4 & 103,9 & 47,3 & 66,7 & 86,6 & 56,8 & 78,3 & 44 & 40,7 & 71,5 & 27,7 \\
\hline
\end{tabular}

Fuente: Anuarios Estadísticos de la Comisión Económicos para América Latina (CEPAL). 1995 y 1990.

Defunciones por causa: OPS. Estadísticas de Salud de las Américas (publicación científica 556) 1995.

Nacimientos:CELADE. Estimaciones y Proyecciones de Población en los países de América Latina 1950-2050

Boletín Demográfico 58. 1996.

Crecimiento del Consumo de Energía. Banco Mundial.

IDH: Indice de Desarrollo Humano. Naciones Unidas 1997.

Tasa acumulada de crecimiento del PIB 1980-87 en \$ U.S.de 1986: Inter-American Development Bank:

Social and Economic Progress in Latin America, 1987.

ricos, en 1994, acumulan el 31\% del ingreso total del país, mientras que, en 1980, ese porcentaje era de 10 puntos menos), seguido de México, con $37 \%$ de aumento, y Panamá, con $28 \%$.

El indicador de desigualdad del ingreso, por un lado, muestra que las diferencias del ingreso aumentaron entre los hogares más ricos y los más pobres, entre 1980 y 1986. Es decir, los ricos reciben mayor proporción del ingreso, en detrimento de lo poco ya acumulado por los pobres.

En 1980, el de mayor desigualdad en la acumulación del ingreso es Colombia y, en segun- do lugar, Brasil (el 10\% de los hogares más ricos acumulan 12 y 10 veces más que los $20 \%$ más pobres). En Panamá, esa desigualdad es de 6 veces, en Argentina y Uruguay, es de 4,5 veces $y$, en Costa Rica y Venezuela, esa desigualdad es alrededor de 3,2. Ya para 1986 el índice de desigualdad considerado disminuye en Colombia y se estabiliza en Uruguay. Brasil acentúa la ya amplia desigual dad (en 1986, pasa a casi 15 veces el ingreso del 10\% más rico en relación al $20 \%$ más pobre) y casi duplica en Venezuela (pasando a ser cinco veces ese cociente de de- 
sigualdad). A pesar de no disponer de la información para México, en 1980, se aprecia que la desigualdad duplica entre 1986 y 1990 (pasando de 3 a 6).

Mas, por otra parte, al contrario de la acumulación de la riqueza, a partir de 1986 se estabiliza el promedio de la desigualdad en los países estudiados. Ello se debe a que algunos países, como Brasil, Colombia y Uruguay, disminuyen el grado de desigualdad, mientras que otros, como Argentina, México y Panamá, continuaron ampliando la brecha de la desigualdad entre los pobres y los ricos. Al final del período considerado, se tienen amplias diferencias entre los niveles de desigualdad de los países.

La otra mudanza importante se da entre 1990 y 1994. Se destaca de nuevo Brasil, por conseguir una reducción de la desigualdad, colocándose en el nivel similar al que tenía en 1980. Por el contrario, Colombia, después de reducir la desigualdad en los años ochenta, se la acentúa en el primer quinquenio de los noventa, por lo que llega a un cociente de desigualdad de 10 y 11 veces, respectivamente.

Aunque las cifras sobre gastos del gobierno no son directamente comparables entre países (por las diferencias de concepción del gasto público), es posible visualizar que, aunque la proporción del gasto público en salud no disminuyó significativamente entre los países comparados y hasta aumentó en países como Argentina, Brasil y México, sigue siendo poco lo que se destina a una de las principales víctimas del crecimiento de la pobreza y de la reducción del poder adquisitivo de la población, la salud. Llama una vez más la atención que, siendo Venezuela de los países con mayor deterioro de la calidad de vida de sus habitantes, no sólo es el único que no aumenta el gasto en salud, sino que, además, es el que se mantiene con la menor proporción de gasto público en salud (apenas $1,3 \%)$.

Evolución temporal de la mortalidad infantil

Comparando el CMI entre los países latinoamericanos seleccionados, se encuentra que, si bien es cierto que se redujo, en todos los países latinoamericanos, durante el período de crisis desde los años ochenta, esas reducciones son diferenciales para cada uno de los países. En el último período estudiado (1990-95), existe gran amplitud del CMI, desde 14 para Chile y Costa Rica a 47 para Brasil. De hecho, es la variable con mayor desvío padrón.

El orden en el nivel del CMI de los países estudiados varía entre las tres décadas observadas. En el primer quinquenio de los setenta, los tres países con menor CMI son Panamá, Uruguay y Venezuela (ente 42 y 48 defunciones de menores de un año por mil nacidos vivos). Costa Rica y Chile ocupan el quinto y el sexto lugar, respectivamente. En el segundo quinquenio de los ochenta, la disminución es de $25 \%$ para el promedio de los países en general. Los cambios más importantes en ese orden se deben a la acelerada reducción del CMI en Costa Rica ( $50 \%$ de reducción), a tal punto que pasa a ocupar el primer lugar en menor mortalidad infantil. El resto de los países continúa en una posición similar, a excepción de Uruguay que tiene una acentuada pérdida relativa (desciende para el quinto lugar).

Las mudanzas más importantes se dan en el primer quinquenio de los ochenta, es decir, la reducción del CMI fue diferencial a tal punto que muda la posición relativa que ocupan los países en relación al nivel de MI. Chile alcanza la mayor reducción del período (en un $64 \%$, entre el segundo quinquenio de los años setenta y el primero de los ochenta). Así, Costa Rica, Chile y Argentina se consolidan (durante el período observado) como de menor nivel del CMI (primer, segundo y cuarto lugar, respectivamente).

En la Figura 1, se observa que, hasta 1985, hay una gran queda del CMI para la mayoría de los países de la región. A partir de ese momento, Ias curvas muestran menor declive, aproximándose a la estabilidad. Por otra parte, se observan países con amplio deterioro en su posición en la región durante el primer quinquenio de los ochenta, como es el caso de Panamá y Venezuela (apenas decrece su CMI en alrededor de 15\%). Por ello, el primero pasa, del primer lugar a inicios de los setenta a ocupar el quinto a mediados de los ochenta y comienzo de los noventa. En la Figura 1, se nota la relativa estabilidad del CMI en Venezuela, por lo cual desciende al sexto lugar de la región.

Aún cuando el desvío padrón del CMI disminuya consecutivamente, así como ocurre con la fecundidad y el analfabetismo, continua siendo una variable que marca diferencias entre los países. Es decir, si bien es cierto que, desde 1970, se reducen las diferencias entre los países considerados (Tabla 1 ) en relación al CMI, el desvío padrón es de los más altos entre las variables consideradas.

A pesar de las conocidas omisiones de registro de las causas de mortalidad infantil, especialmente en aquellos lugares de calidad de vida más precaria, en donde es más factible la mortalidad por diarrea y otras relacionadas con nutrición e higiene, los CMI por causas infecciosas, entre los distintos países considerados, dan idea, más que de la magnitud del proble- 
Figura 1

Cociente de mortalidad infantil en algunos países latinoamericanos, por quinquenio, de 1975 a 1995.

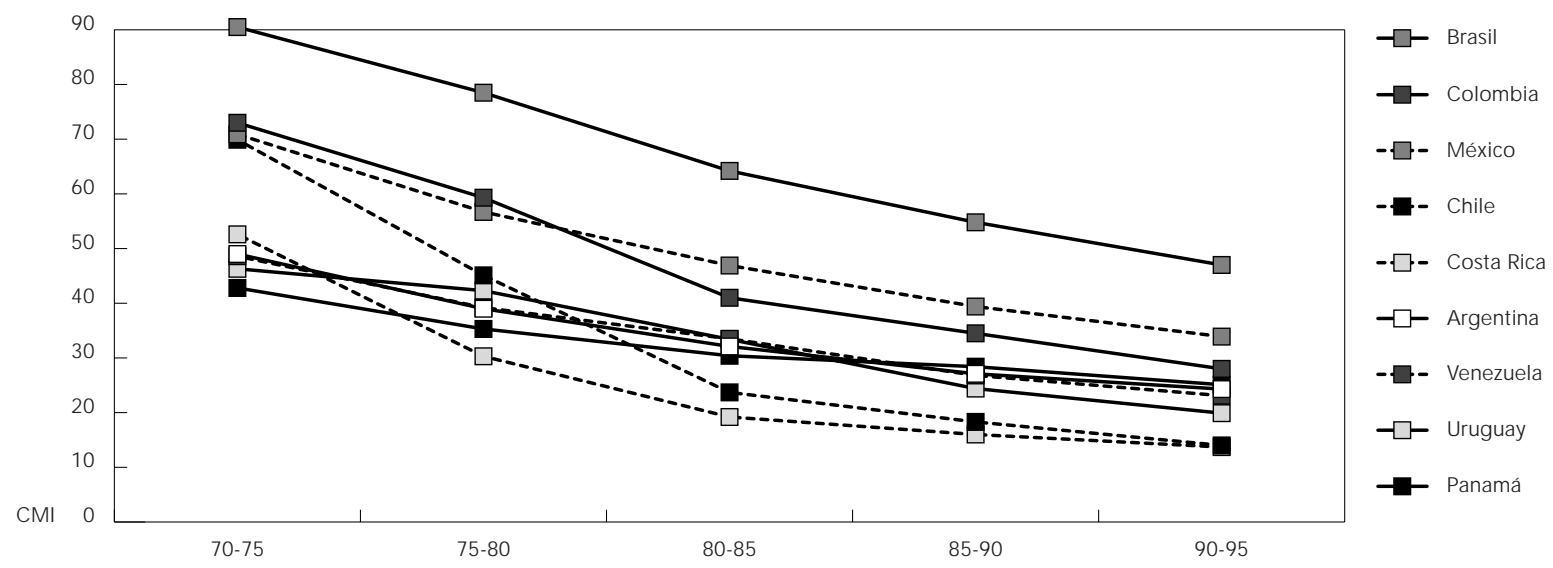

ma, de la intensidad del control de esas causas de mortalidad y de los diferenciales entre los países latinoamericanos considerados para los años 1970, 1980 y 1989. Gracias especialmente a las medidas de saneamiento implantadas, se logró controlar en gran parte la mortalidad por diarrea y otras causas infecciosas, siendo que el promedio para la región pasa de 10 muertes en 1970 a dos muertes por cada mil nacidos vivos en 1990. La desviación entre el CMI por causas infecciosas entre ese promedio y el de cada país también disminuye acentuadamente, es decir, la reducción fue mayor en aquellos países que presentaban mayores tasas (con excepción de México). Mas los de mayor ganancia en la lucha contra ese grupo de causas fueron Chile y Costa Rica (una reducción de 37 y 18 veces, respectivamente, del valor de 1970). La menor reducción del CMI por infección se dio en Venezuela; por ello, mientras que, en 1970, sólo perdía de Uruguay y Panamá, en 1990, pasa a tener una de las más altas mortalidades por causas infecciosas, superada apenas por M éxico y Brasil. A través de esos resultados, se aprecia la pérdida relativa de Venezuela en relación a las ganancias de salud de la región latinoamericana.

La crisis socioeconómica y la mortalidad infantil

En la Figura 2, se muestra el promedio general (expresado en la forma de logaritmo) para la región, del CMI, la Tasa Global de Fecundidad, el analfabetismo, la desigualdad y la pobreza entre 1975 y 1990-95. A pesar de reconocer que ese promedio no expresa las variaciones de los países seleccionados para la comparación, además de no ser ponderados por el tamaño de la población, se lo utiliza como forma de visualizar la tendencia general de los indicadores. En esa figura, es interesante observar que el analfabetismo y la TGF conforman dos rectas casi paralelas, es decir, el ritmo de disminución es similar. En segundo lugar, el decrecimiento del CMI se diferenció de las anteriores variables en los años setenta mas, debido a la disminución en la velocidad de la reducción, a partir de los ochenta comienzan a asemejarse más sus tendencias.

Lo tercero a ser considerado, y quizá más relevante, es que, aunque la TMI continúe en descenso en período de aumento de la pobreza, es justamente a partir de 1985 que el CMI más reduce su descenso y la pobreza aumenta con mayor velocidad. Es decir, si bien es cierto que el CMI continuo disminuyendo en períodos de crisis, fue con menor intensidad, especialmente cuando aumenta la pobreza. Por otra parte, podría afirmarse que, hasta finales de los ochenta, el crecimiento del gasto en salud es similar al del CMI, mas, a partir de 1990, el aumento del primero no acompaña el ritmo del CMI.

El análisis de correlación entre el CMI de 1980-85, mostrado en la Tabla 2, confirma varias de las explicaciones antes señaladas. Como ha sido ampliamente señalado, el analfabe- 
Figura 2

Promedio de los países latinoamericanos en estudio de algunos indicadores, por quinquenio, de 1975 a 1995.

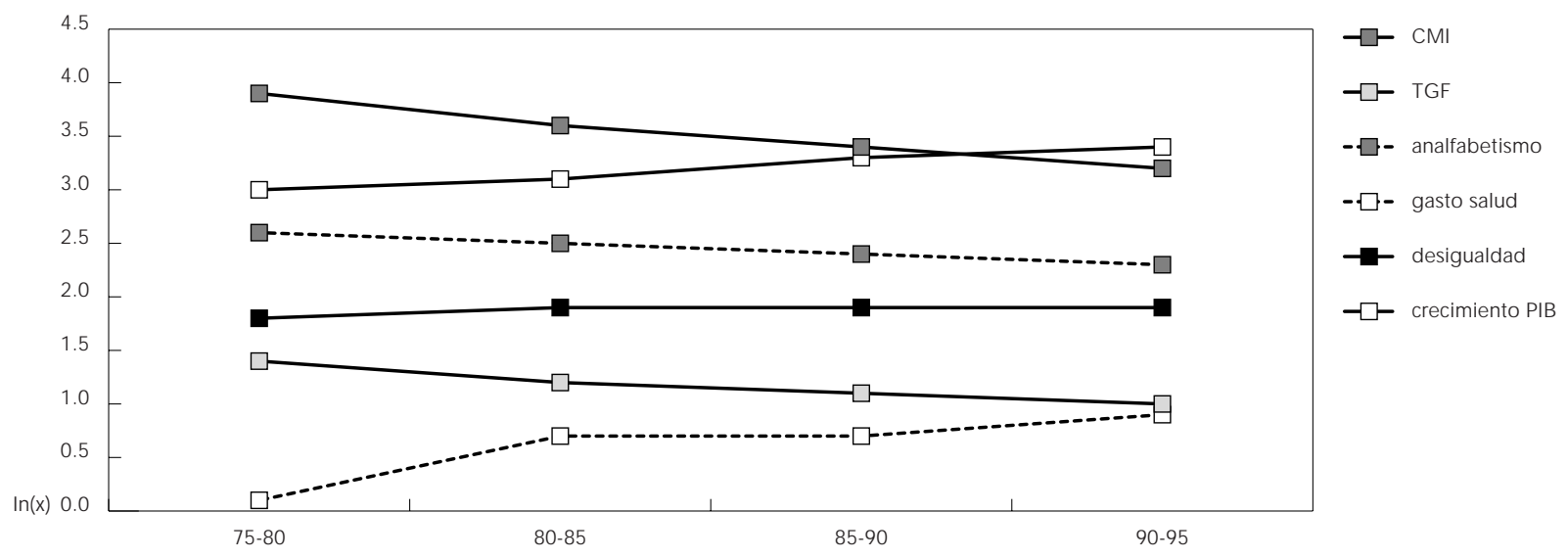

$\mathrm{CMI}=$ Cociente de Mortalidad Infantil; TGF = Tasa Global de Fecundidad; PIB = Producto Interno Bruto.

tismo es el factor más asociado con el CMI (con una correlación de 0,834 y significativo en el nivel de 5\%), es decir, países con menor nivel de analfabetismo tienen menor proporción de fallecidos de menores de un año. Mas, también se encontró que no sólo la pobreza guarda una estrecha relación con los niveles del CMI ( $r=$ 0,677 y $p=0,065$ ) sino que, además, está más asociada con el nivel de pobreza alcanzado en los años setenta que con el de los mismos años de la observación.

Las diferencias entre los países del CMI de 1980-85 y el crecimiento positivo en la mayoría de los países latinoamericanos del PIB por habitante, entre 1965 y 1980, tienen una relación significativa $(r=0,668$ y $p=0,049)$, mientras que el decrecimiento generalizado del PIB durante los ochenta no guarda ninguna relación.

La desigualdad en 1980 aparece como la segunda variable con más alta correlación (significativo a un nivel de 10\%) con el CMI entre el conjunto de países latinoamericanos sobre análisis, mas debe tenerse en cuenta que el número de datos observados es menor $(n=7)$.

Aunque la relación con el CMI no sea significativa en el nivel de $10 \%$, los resultados en relación al gasto en salud (tanto de 1980 como de 1985) y el porcentaje de población en área metropolitana muestran una relación inversa con el CMI. Tomando como referencia 1985, se tiene que Costa Rica, con la mayor proporción del gasto del gobierno en el área de salud, tiene el menor CMI. Situación contraria a la de Colombia y México, con menos concentración urba- na y menor gasto en salud. Llama la atención el caso de Brasil, el cual, con el mayor CMI, tiene una proporción de gasto en salud similar al de Chile. También hay que considerar que Brasil es el de proporción de población en area metropolitana.

Debido a que la Tasa Global de Fecundidad cada vez más es similar, entre los distintos países considerados, como puede ser observado por el desvío padrón, no presenta una correlación significativa con los CMI. Mas, se constata su relación directa con la MI, sobre todo la alcanzada en el quinquenio anterior al CMI observado.

A continuación, se comparan la velocidad del ritmo de decrecimiento del CMI, la pobreza y el PIB en dos períodos, que representan de alguna manera lo acontecido antes (o comienzo) de la crisis y después de la instauración de la misma. En la Tabla 3 y la Figura 3, se presentan la variación porcentual del decrecimiento del $\mathrm{CMI}$ entre el segundo quinquenio de los setenta y el primero de los ochenta y la variación porcentual del decrecimiento del CMI que ocurrió entre finales de los años ochenta y primer quinquenio de los noventa. Además, en la Tabla 3 y en la Figura 4, se encuentran Ias variaciones porcentuales del crecimiento de la pobreza de 1970 a 1980 y entre 1980 y 1990.

Se verifican la pérdida en el ritmo de decrecimiento del CMI y el aumento de la variación porcentual de la pobreza en los años ochenta. En otras palabras, aunque el CMI continúe en descenso lo hace con una velocidad menor, in- 
Correlación de Pearson entre las variables socio-económicas y la tasa de mortalidad infantil de quinquenio 1980-1985 en el grupo de países latinoamericanos seleccionados.

\begin{tabular}{lccc}
\hline & Correlación ( $r)$ & Significancia $(p)$ & No de países \\
\hline Analfabetismo 1980 & 0,8342 & 0,005 & 9 \\
Pobreza 1970 & 0,6771 & 0,065 & 8 \\
Desigualdad 1980 & 0,7001 & 0,080 & 7 \\
Desigualdad 1986 & 0,5841 & 0,098 & 9 \\
Crecimiento del PIB 1965-80 & 0,6682 & 0,049 & 9 \\
Gasto en salud 1980 & $-0,563$ & 0,114 & 9 \\
Gasto en salud 1985 & $-0,511$ & 0,160 & 9 \\
Población en área metropolitana 1980 & $-0,506$ & 0,164 & 9 \\
TGF 1975-80 & 0,499 & 0,171 & 9 \\
TGF 1980-85 & 0,391 & 0,298 & 9 \\
Pobreza 1980 & 0,150 & 0,699 & 9 \\
Crecimineto del PIB 1980-85 & 0,159 & 0,682 & 9 \\
\hline
\end{tabular}

1 Significativo al nivel de $10 \%$.

2 Significativo al nivel de $5 \%$.

Tabla 3

Diferencia porcentual entre el crecimiento del coeficiente de mortalidad infantil y de la pobreza.

\begin{tabular}{lcccc}
\hline & \multicolumn{2}{c}{ Crecimiento del CMI } & \multicolumn{2}{c}{ Crecimiento de la pobreza } \\
& $1975-80$ a $1980-85$ & $1985-90$ a $1990-95$ & 1970 a 1980 & 1980 a 1990 \\
\hline Argentina & $-21,5$ & $-11,5$ & 40,0 & 171,4 \\
Brasil & $-22,3$ & $-16,6$ & $-14,3$ & 30,0 \\
Chile & $-90,3$ & $-30,7$ & 241,7 & $-17,1$ \\
Colombia & $-44,6$ & $-23,2$ & $-5,3$ & $-2,8$ \\
Costa Rica & $-57,8$ & $-16,8$ & 6,7 & 37,5 \\
México & $-20,9$ & $-16,2$ & 0,0 & 70,0 \\
Panamá & $-16,1$ & $-13,1$ & - & 9,7 \\
Uruguay & $-26,6$ & $-22,6$ & $-10,0$ & $-10,0$ \\
Venezuela & $-17,0$ & $-15,9$ & $-10,1$ \\
\hline
\end{tabular}

clusive en aquellos países con niveles más altos como Brasil, Colombia, M éxico y Argentina. En relación a la pobreza, se observa no sólo que, en los años setenta, disminuyó su crecimiento, mientras que, en los noventa, creció aceleradamente. Además, los países con mayor aumento del ritmo de crecimiento de la pobreza son, en su mayoría, los de menor variación porcentual en el CMI de finales de los ochenta y principios de los noventa.

En la Tabla 3, también puede apreciarse que, a principio de los ochenta, países que ya habían alcanzado los más bajos niveles del CMI en latinoamérica y que también pasaban por crisis económica, tales como Chile, Uru- guay y Costa Rica, tienen así mismo mayor decrecimiento del CMI. Los países con mayor CMI y, por tanto, con mayor proporción de causas de muertes infantiles de fácil erradicación con medidas básicas de saneamiento son los de mayor reducción en el decrecimiento del CMI.

Se estimó la correlación entre las diferencias de la variación porcentual del crecimiento del CMI y las diferencias en el crecimiento de la pobreza en esos dos períodos señalados en la Tabla 3 y el crecimiento interanual del PIB entre 1965 y 1980 y de 1980 a 1989 (presentado en la Tabla 1). Los cambios en el ritmo de decrecimiento del CMI tienen una correlación 


\section{Figura 3}

Variaciones porcentuales del decrécimo del coeficiente de mortalidad infantil de 1975-80 a 1980-85 y de $1985-90$ a 1990-95.

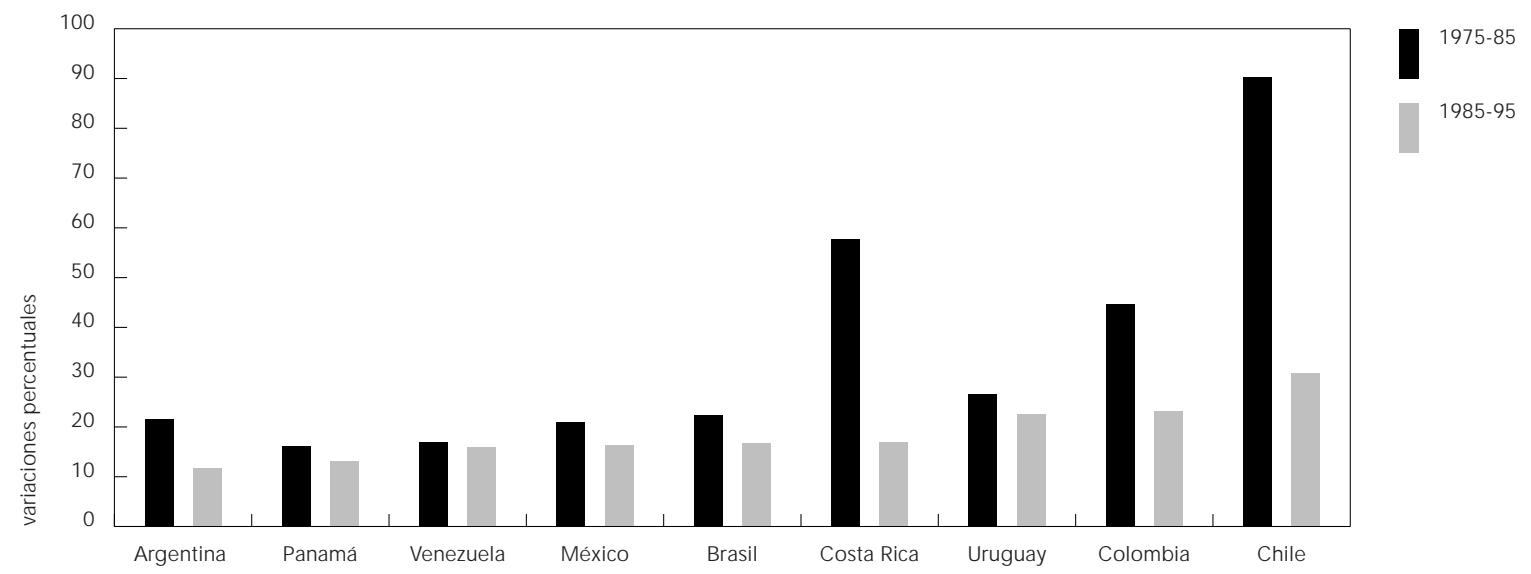

\section{Figura 4}

Variaciones porcentuales del crecimiento de la pobreza de 1970 a 1980 y de 1980 a 1990.

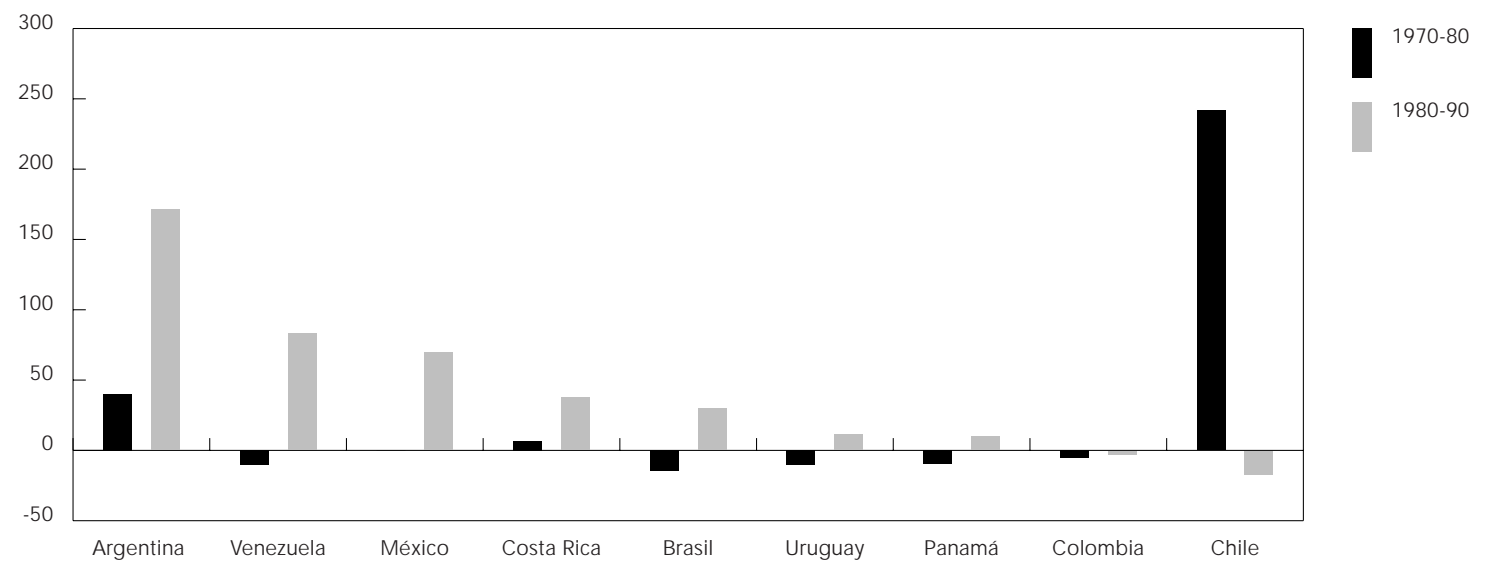

negativa de 0,699 ( $p=0,054)$ con las diferencias en las variaciones porcentuales de la pobreza y una positiva ( $r=0,6058$ y $p=0,084)$ con las variaciones de crecimiento del PIB. Ello muestra que los países con mayores disminuciones en el crecimiento del PIB y mayores aumentos de la pobreza son aquellos que presentaron mayor desaceleración en el declino del CMI, tales como Argentina, Colombia y Brasil.

\section{Discusión}

El Cociente de Mortalidad Infantil es conocido como uno de los más sensibles y comunes indicadores del desarrollo social y económico de una población (Woodbury, 1925; Stockwell, 1962; Behm, 1979; Breilh \& Granda, 1984; Mosley \& Chen, 1984; Masuy-Stroobant \& Gourbin, 1995). Mas, si bien es cierto que no es fácil me- 
dir esa sensibilidad en períodos cortos, como lo señalan Palloni \& Tienda (1992), es importante continuar demostrando que los patrones demográficos generalmente no son inmunes a fluctuaciones económicas.

En este trabajo, realizado a nivel agregado y con información de nueve países de América Latina, se encontraron evidencias de la asociación entre crisis económica y el Cociente de Mortalidad Infantil en la década de los ochenta, los cuales, más que conclusiones definitivas, traen algunas cuestiones para la reflexión. Sobre todo porque la crisis económica que comenzó en la llamada década perdida no sólo no acabó, sino que, por el contrario, se ha arraigado en cambios estructurales de la sociedad.

En primer lugar, si bien es cierto que el CMI continuó en descenso, no puede sustentarse que su tendencia reciente pueda desvincularse de los efectos particulares de la crisis económica desatada desde principios de los años ochenta. Los resultados de este trabajo confirman que la mortalidad infantil está asociada negativamente con las mudanzas económicas de corto plazo. Más específicamente, se observó una correlación negativa y significativa entre el ritmo de decrecimiento del CMI y el crecimiento de la pobreza. Además que es justamente a partir de la mayor acentuación de la crisis en 1985 cuando mayor relación se tiene entre la más lenta disminución del CMI y el acelerado aumento de la pobreza.

En ese sentido, Hill y Palloni (1994) contrastaron la tendencia del CMI con las oscilaciones económicas, usando como variable mensuradora de éstas el consumo personal en términos reales. Encontraron coeficientes estadísticamente poco significativos, mas sistemáticos y consistentes, que muestran que la mortalidad infantil está asociada negativamente con las mudanzas económicas de años cercanos. Ja Bronfman (1992) mostró que el impacto de la crisis mexicana sobre la mortalidad infantil refleja no un cambio de rumbo de la tendencia a la disminución, mas sí un incremento en la desigualdad social.

Por otra parte, en este trabajo se mostró que el impacto de la crisis económica de los ochenta tuvo diferente intensidad entre los países considerados. Panamá, Venezuela y Uruguay son los que más mudan su posición relativamente ventajosa alcanzada en los años setenta en lo que se refiere a la situación de la mortalidad infantil entre los países de la región.

Una de las cuestiones interesantes para incluir en esta discusión sería el intentar explicar por qué de esas diferencias en la intensidad del efecto entre paísses que pasaron por similar crisis económica. En cuanto a los casos específicos de Chile y Costa Rica (los cuales, junto con Cuba, se señalan como ejemplos de diferentes contextos socioeconómicos con mejoras significativas de la situación materno-infantil, aún en períodos de crisis), Taucher (1988) ha mencionado que la postergación de los matrimonios y nacimientos en períodos de dificultades económicas ha reducido los riesgos de mortalidad al nacimiento (sobre todo los nacimientos de madres adultas y la alta paridad). Por otra parte, Bravo (1997) agrega a esas explicaciones el aumento del acceso a los servicios primarios de salud, la efectiva localización de recursos de esos programas y la mejora del nivel educativo.

Adicionalmente, otra hipótesis para explicar las diferencias en el impacto de la crisis económica entre los países latinoamericanos está relacionada con el patrón demográfico presente en el momento de la crisis (Beltrão \& Sawyer, 1990; Lee, 1990; Legrand \& Phillips, 1996). No sólo la estructura por edad y los componentes de la fecundidad (edad de la madre al parto, intervalo intergenésico y orden de nacimiento), que pueden mediar la influencia de la crisis en la mortalidad infantil (Hobcraft et al., 1985), sino además, por el tipo e intensidad de desplazamiento migratorio (por ejemplo, rural-urbano, la migración de retorno, como señala Palloni, 1990).

Una de las hipótesis más consideradas en este contexto es la que incorpora el papel de la educación materna (Das Gupta, 1997a) y el papel de las políticas de salud pública. Algunos autores consideran que es posible pensar que, si bien es cierto que ha declinado el gasto en salud pública, es posible esperar en algunos países mejoras en la eficiencia de administración de los reducidos fondos como, por ejemplo, la proporción destinada a la prevención (Bravo, 1997; Musgrove, 1997). Además, el tipo de programa adoptado (objetivos, población destinataria, temporalidad) también afectaría a la disminución del CMI (Palloni, 1985).

En los resultados de este estudio se tiene que el aumento del gasto en salud de los años setenta coincide con la mayor reducción del CMI, se estabilizan esas dos variables en los ochenta, mientras que, a comienzos de los noventa, la reducción del decrecimiento del CMI se da a pesar del aumento del gasto en salud en algunos países. Eso podría deberse a las diferencias de la eficiencia y eficacia del gasto en salud entre los períodos considerados. Mas también hay que tomar en cuenta que el indicador de gasto en salud utilizado no es homogéneo en su definición. Por lo tanto, sería importante generar indicadores de mayor veraci- 
dad y comparabilidad entre los países, en relación al tema de gasto en salud.

Mas, por otro lado, también es importante considerar que el deterioro de los servicios de salud no necesariamente llevaría al aumento directo de la morbilidad y de la mortalidad infantil, ya que existirían estrategias a nivel colectivo (como la ayuda entre las familias y los vecinos para compartir la vivienda y el cuidado de los niños) y a nivel de organización local (como programas alimentarios) que podrían solventar la pérdida del ingreso del hogar y de la calidad de vida (Das Gupta, 1989; Palloni \& Tienda, 1992).

En el presente trabajo, una de las hipótesis que más se torna explicativa de las diferencias en los efectos que dicen respecto a las variaciones del descenso del CMI es el impacto de la desigualdad del ingreso acentuada por la particular crisis económica desde los años ochenta. En 1960, el 20\% de la población mundial que vivía en los países más ricos tenía 30 veces el ingreso del $20 \%$ más pobre y, en 1995, tenía 82 veces ese ingreso. La enormidad de la riqueza de los ricos es un contraste chocante con los bajos ingresos del mundo en desarrollo. Se estima que menos del $4 \%$ de la riqueza combinada de las 225 personas más ricas del mundo sería suficiente para lograr el acceso universal a los servicios básicos en todo el mundo (Naciones Unidas, 1998).

Tomando en consideración las limitaciones impuestas por el reducido número de países considerados en este estudio, una observación importante obtenida con el análisis de correlación efectuado es que la desigualdad, después del nivel de analfabetismo, es la variable con mayor relación con el CMI. Ello confirma lo observado recientemente por Kawachi \& Kennedy (1997) en el sentido que sociedades con mayor concentración del ingreso son las que menos invierten en efectivas políticas sociales, en educación, vivienda y asistencia médica pública.

Todavía, hay que considerar que el retroceso en la escala del ingreso de amplios sectores urbanos de clase media, y las dificultades para su recuperación a corto plazo, dada la escasez de empleos y los bajos salarios ofrecidos, es considerado como de implicaciones relevantes de la crisis económica de los ochenta (Bustelo, 1994; Massey, 1996). Las políticas de ajustes que están siendo implantadas no incorporan a esos "nuevos pobres", Ios cuales han ido perdiendo privilegios obtenidos en décadas anteriores (como seguro social, escuelas públicas de calidad, estabilidad laboral, entre otras). De esa forma, se está creando una estructura social en la cual muchos podrán quedar excluidos (Bustelo, 1994).
Como señala Massey (1996), después de los años setenta, la promesa de movilidad social masificada se evaporó, y la desigualdad marcó una nueva era en donde los privilegios de los ricos y las desventajas de los pobres se incrementaron, a la par de las mudanzas en la estructura geográfica de la desigualdad. Si la hipótesis de Massey (1996) se constata, donde el nuevo orden espacial dominante concentre y separe cada vez más la pobreza y la riqueza, se tendrán profundas implicaciones en la vida social e individual, oponiendo aún más sub-culturas, valores, acceso a los servicios (entre otros). Una de las consecuencias hasta ahora más visible es la proliferación de la violencia (AnzolaPerez \& Bangdiwala, 1996; Szwarcwald \& et al., 1999) mas, con el tiempo, se podrán ver acentuadas otras mudanzas sociales. Por otra parte, la oposición de las subculturas entre ricos y pobres puede conllevar a disminuir el acceso a la información y la formación de patrones de referencia de los hogares más pobres en relación a la atención de la salud materno-infantil, factores considerados importantes para extender las habilidades básicas para cuidado de los hijos, independiente del status, de la ocupación y de la escolaridad de la madre (Das Gupta, 1989).

Otra posible consecuencia de la concentración residencial de la pobreza es la aglomeración de las muertes infantiles en un pequeño número de familias (madres con más de un hijo fallecido en la infancia), en otra palabras, comunidades carentes concentrando un gran número de niños fallecidos (Bronfman, 1993; Das Gupta, 1997b). Si, por un lado, esa concentración de decesos poco afecta a la tendencia general del descenso del CMI, por otro, la desagregación geográfica mostrará patrones bien diferenciados de niveles y estructura por causa de la mortalidad infantil.

I gualmente, el hecho de encontrar en este trabajo que el grado de urbanización no tenga una alta relación con el nivel del CMI puedeestar asociado con la reorganización espacial, como consecuencia del patrón de desigualdad que se está arraigando con el nuevo orden económico y social (Massey, 1996). En otras palabras, puede estar mudando el efecto positivo que tenía el crecimiento de las ciudades, y con ello la escolaridad, en la mejora de la prevención y atención de la salud, especialmente por la concentración de recursos hospitalarios y de acceso a los servicios públicos que se dio en las décadas de los sesenta y setenta.

Para finalizar, es importante reafirmar la necesidad de más estudios sobre los cambios de la mortalidad infantil en períodos de corto 
plazo e incorporar niveles de mayor desagregación de la información ya que, como señalan Palloni \& Tienda (1992), es preciso comparar estimaciones del CMI diferenciados por status social, para observar claramente el efecto de las recesiones económicas, de lo contrario, sólo será visible y evidenciado en países con una paupérrima calidad de vida y con desigualdad social. Ese tipo de estudio es importante sobre todo en la región latinoamericana, la cual no sólo es la región de mayor desigualdad en el

\section{Agradecimientos}

La investigación en que se basa este trabajo contó con el apoyo financiero de la Fundação de Amparo a Pesquisa do Estado do Rio de Janeiro (FAPERJ).

\section{Referencias}

ANZOLA-PEREZ, E. \& BANGDIWALA, S. I., 1996. The changing structure of deaths form injuries and violence. In: Adult Mortality in Latin America (I. M. Timateus, J. Chackiel \& L. Ruzicka, eds.), pp. 306336, Oxford: Claredon Press.

BAJ RAJ, R. \& BRAVO, J., 1993. Una visión sintética del ajuste económico y sus consecuencias demográficas en América Latina. Notas de Población, 59: 51-72.

BEHM, H., 1979. Socio-economic determinants of mortality in Latin America. In: Proceedings of the Meeting on Socioeconomic Determinants and Consequences of Mortality, pp. 140-165, Geneva: World Health Organization.

BELTRÃO, K. \& SAWYER, D., 1990. Medidas de Mortalidade: Um Estudo sobre os Efeitos das Mudanças da Escolaridade da Mãe e da Estrutura da Fecundidade em Quatro Áreas Brasileiras. Rio de Janeiro: Escola Nacional de Ciências Estatísticas/ Fundação Instituto Brasileiro de Geografia e Estatística.

BRAVO, J., 1997. Demographic consequences of economic adjustment in Chile. In: Demographic Responses to Economic Adjustment in Latin America (G. Tapinos, A. Mason \& J. Bravo, eds.), pp. 156173, Oxford: Claredon Press.

BRAVO, J. \& RODRIGUEZ, V., 1993. Análisis de las fluctuaciones demográficas a corto plazo: Una visión estadística de las anotaciones demográficas de Raúl Prebisch. Notas de Población, 58:9-29.

BREILH, J. \& GRANDA, E., 1984. Un marco teórico sobre los determinantes de la mortalidad. In: Memorias del Congreso Latinoamericano de Población (Programa de Investigaciones Sociales sobre Población en América Latina, v. 1), pp. 131-156, México, D.F.: Universidad Nacional Autónoma de México/El Colegio de México.

BRONFMAN, M., 1992. Infant mortality and crisis in Mexico. International Journal of Health Services, 22:157-167. mundo sino, además, aquella cuya distribución del ingreso más empeoró durante la década de los ochenta (CEPAL, 1993; World Bank, 1993). Mismo cuando las economías tienden a recuperarse y hasta cuando se disminuye la pobreza, la desigual dad no acompaña con la misma intensidad esa tendencia (Bustelo, 1994) y, por lo tanto, los eventos asociados a esa desigualdad, como la mortalidad infantil, no pueden revertir rápidamente las pérdidas ocasionadas por la crisis económica.
BRONFMAN, M., 1993. Multimortalidad y Estructura Familiar: Un Estudio Cualitativo de las Muertes Infantiles en las Familias. Tese de Doutorado, Rio de Janeiro: Escola Nacional de Saúde Pública, Fundação Oswaldo Cruz.

BUSTELO, E., 1994. Hood Robin: Ajuste e eqüidade na América Latina. Planejamento e Políticas Públicas, 11:5-48

CELADE (Centro Latinoamericano de Demografía), 1996. Estimaciones y Proyecciones de Población en Ios Países de América Latina 1950-2050. Boletín Demográfico no 58. Santiago: CELADE.

CEPAL (Comisión Económica para América Latina y el Caribe), 1990. Anuarios Estadísticos deAmérica Latina y el Caribe. Santiago: CEPAL.

CEPAL (Comisión Económica para América Latina y el Caribe), 1993. La Equidad en el Panorama Social de América Latina durante los Años Ochenta. Santiago: CEPAL.

CEPAL (Comisión Económica para América Latina y el Caribe), 1995. Anuarios Estadísticos de América Latina y el Caribe. Santiago: CEPAL.

CHACKIEL, J., 1984. La mortalidad en América Latina: Niveles, tendencias y determinantes. In: $\mathrm{Me}$ morias del Congreso Latinoamericano de Población (Programa de Investigaciones Sociales sobre Población en América Latina, v. 1), pp. 157-186, México, D.F.: Universidad Nacional Autónoma de México/El Colegio de México.

CORNIA, G. \& FRANCES, S., 1990. Sistema fiscal, ajuste y pobreza. Colección Estudios Corporación de Investigación Económica para Latinoamérica (CIEPLAN), 31:77-106.

DAS GUPTA, M., 1989. Death Clustering, Maternal Education and the Determinants of Child Mortality in Rural Punjab, India. Center for Population Studies Discussion Paper no 89-1. Cambridge: Center for Population Studies, Harvard University.

DAS GUPTA, M., 1997a. Socio-economic status and clustering of child deaths in rural Punjab. Popu- 
Iation Studies, 51:191-202.

DAS GUPTA, M., 1997b. Methodology and main findings on child survival of the Khanna restudy, 1984-1988. In: Prospective Community Studies in Developing Countries (M. Das Gupta, ed.), pp. 81100, Oxford: Clarendon Press/ Liège: International Union for the Scientific Study of Population.

HILL, K. \& PALLONI, A., 1994. Demographic responses to economic shocks: The case of Latin America. Research in Human Capital and Development, 8:197-223.

HOBCRAFT, J.; MCDONALD, J. W. \& RUTSTEIN, S. O. 1985. Demographic determinants of infant and early child mortality: A comparative analysis. Population Studies, 39:363-385.

KAWACHI, I. \& KENNEDY, B. P., 1997. The relationship of income inequality to mortality: Does the choice of indicator matter? Social Science and Medicine, 45:1121-1127.

LEE, R., 1990. La reacción demográfica ante las crisis económicas en poblaciones históricas y contemporáneas. Boletín delas Naciones Unidas, 29:1-15.

LEGRAND, T. \& PHILLIPS, J., 1996. The effect of fertility reductions on infant and child mortality: Evidence from Matlab in rural Bangladesh. Population Studies, 50:51-68.

M ASSEY, D. S., 1996. The age of extremes: Concentrated affluence and poverty in the twenty-first century. Demography, 33:395-412.

MASUY-STROOBANT, G. \& GOURBIN, C., 1995. Infant health and mortality indicators. European Journal of Population, 11:63-84.

MOSLEY, W. \& CHEN, L. C., 1984. An analytical framework for the study of child survival in developing countries. Population and Development Review, 10 (Sup.):25-45.

MUSGROVE, P., 1997. Economic crisis and health policy response. In: Demographic Responses to Economic Adjustment in Latin America (G. Tapinos, A. Mason \& J. Bravo, eds.), pp. 37-53, Oxford: Claredon Press.

NACIONES UNIDAS, 1998. Informe sobre Desarrollo Humano 1998. Madrid: Mundi-Prensa Libros.

OLIVEIRA, L. \& SIM ÕES, C., 1986. O papel das políticas de saúde e saneamento na recente queda da mortalidade infantil: Significado, alcance e limitações estruturais. In: Perfil Estatístico de Crianças e Mães no Brasil. Aspectos Sócio-Econômicos da Mortalidade Infantil em Áreas Urbanas, pp. 51-57, Rio de Janeiro: Fundação Instituto Brasileiro de Geografia e Estatística/ Fundo das Nações Unidas para a Infância.

OPS (Organización Panamericana de la Salud), 1995. Publicación Científica 556. Washington, D.C.: OPS.

PALLONI, A., 1985. Santé et lutte contre la mortalité en Amerique Latine. In: La Lutte Contre la Mort. Influence des Politiques Social es et des Politiques deSantésur l'Évolution dela Mortalité(J. Vallin \& A. Lopez, org.), pp. 447-474, Paris: Presses Universitaires de France.

PALLONI, A., 1990. Assessing the levels and impact of mortality in crisis situations. In: Measurement and Analysis of Mortality (J. Vallin, S. D'Souza \& A. Palloni, eds.), pp. 194-228, Oxford: Claredon Press.

PALLONI, A., 1994. Comentarios a la sesión sobre la dimensión salud en los planteamientos sobre transición demográfica. In: Memorias de Ia IV Conferencia Latinoamericana de Población, v. 1 , pp. 511-520, México, D.F.: Instituto Nacional de Estadística, Geografía e Informática/Instituto de Investigaciones Sociales de la Universidad $\mathrm{Na}$ cional Autónoma de México.

PALLONI, A.; HILL, K. \& AGUIRRE, G. P., 1996. Economic swings and demographic changes in the history of Latin America. Population Studies, 50: 105-132.

PALLONI, A. \& TIENDA, M., 1992. Demographic responses to economic recessions in Latin America since 1900. Sociological Inquiry, 62:246-270.

PETRAS, J. \& VELTMEYER, H., 1995. La recuperación económica de América Latina: El mito y la realidad. Nueva Sociedad, 137:164-179.

RIOS-NETO, E. \& DE CARVALHO, M., 1997. The demographic consequences of structural adjustment: The case of Brazil. In: Demographic Responses to Economic Adjustment in Latin America. (G. Tapinos, A. Mason \&J. Bravo, eds.), pp. 174198, Oxford: Claredon Press.

STOCKWELL, E. G., 1962. Infant mortality and socioeconomic status: A changing relationship. Milbank Memorial Fund Quarterly, 40:101-111.

SZWARCWALD, C. L.; BASTOS, F. I.; VIACAVA, F. \& ANDRADE, C., 1999. Income inequality and homicide rates: A study in Rio de Janeiro, Brazil. American Journal of Public Health, 89:845-850.

SZWARCWALD, C. L.; LEAL, M. C.; CASTILHO, E. A. \& ANDRADE, C. L. T., 1997. Mortalidade infantil no Brasil: Belíndia ou Bulgária? Cadernos de Saúde Pública, 13:503-516.

TAPINOS, G.; MASON, A. \& BRAVO, J., 1997. Demographic Responses to Economic Adjustment in Latin America. Oxford: Claredon Press.

TAUCHER, E., 1988. Efecto del Descenso de la Fecundidad en la Mortalidad Infantil. Estudio Técnico IDRC-TS57s. Bogotá: Centro Internacional de Investigaciones para el Desarrollo.

TOWNSEND, P. \& DAVIDSON, N., 1990. Inequalities in Health. TheBlack Report and the Health Divise London: Penguin.

WILKINSON, R. G., 1992. Nation mortality rates: The impact of inequality? American Journal of Public Health, 82:1082-1084.

WOOD, C. H. \& CARVALHO, J., 1988. The Demography of Inequality in Brazil. New York: Cambridge University Press.

WOODBURY, R. M., 1925. Causal Factors in infant mortality. Washington, D.C.: Government Printing Office.

WORLD BANK, 1991. World Development Report. Oxford: University Press.

WORLD BANK, 1993. Poverty and Income Distribution in Latin America: The Story of the 1980s. Washington, D.C.: Technical Department, Latin America and the Caribbean Region, World Bank.

WORLD BANK, 1996. World Devel opment Report. Oxford: University Press. 